\title{
nature
}

geoscience

\section{Globalizing quake information}

\author{
Destruction from earthquakes continues to threaten poor and wealthy nations alike. The \\ Global Earthquake Model is a potentially important step towards providing risk information \\ on a worldwide basis, using a unified standard.
}

The devastation wreaked by large earthquakes is a stark reminder of how small and vulnerable human beings are when confronted with the forces of nature. The regularity with which the world has been surprised by these forces during the most recent past would seem to vindicate the famous remark by the Pulitzer-prizewinning historian Will Durant: "civilization exists by geologic consent, subject to change without notice". Yet a closer look at the facts suggests that the loss of human life and property was far from inevitable: glaring lacunae in disaster preparedness and longterm planning were at least as responsible as the ferocity of nature.

Although geoscientists cannot predict when the next big earthquake will strike, they have a much better understanding of where quakes are likely to occur and, in some cases, how frequently they have occurred in the past. The Global Earthquake Model (http://www.globalquakemodel.org/) is an open-source initiative aimed at creating the definitive instrument for the calculation and communication of earthquake risks. It is a promising attempt to pool regional knowledge to create one reliable global resource and to facilitate the communication of the results to policy makers and the public.

The Global Earthquake Model is an initiative of the Organisation for Economic Co-operation and Development (OECD), set to launch in early 2009. But work on a technology demonstrator version, $\mathrm{GEM}_{1}$, has already begun. When fully functional, it will be possible to calculate the probability that an earthquake will strike a particular region during a user-specified timeframe, and also the expected ground motion. This capability will be founded on an extensive database of geological and geophysical data, such as the locations of active faults, historical earthquakes, and the nature of soils. The model will be constantly refined as new information becomes available and the estimation of probabilities will be dynamic rather than static.

When combined with data on population density and the quality of buildings, the system will allow the estimation of the likely damage to life and property. End users will be able to ask specific queries and obtain results in the form of maps or tables. For example, homebuyers may request maps showing the seismic hazard for a locality of interest, and local governments or insurance companies may seek maps showing likely casualties and property damage due to an earthquake in a specific region.

\section{Yet a closer look at the facts} suggests that the loss of human life and property was far from inevitable: glaring lacunae in disaster preparedness and long-term planning were at least as responsible as the ferocity of nature.

Importantly, all stakeholders - scientists and engineers, governments and the private sector - will collaborate to achieve this goal. Various insurance companies have already contributed substantially to the project. It is not difficult to see why: earthquakes create enormous financial losses that are often insured. Of course, insurance companies already use sophisticated tools to estimate likely pay-outs, making their experience an important asset of the project. In return, through the Global Earthquake Model, companies will have access to the expertise of the global scientific community and new global datasets.

How effective the Global Earthquake Model will be depends critically on the quality of information used in providing hazard and risk estimates. Rich geological and geophysical data sets are available for some regions of the world such as the United States, but the same is not true for less wealthy and developed regions. Because the Global Earthquake Model will not create new data, the output of its various tools will only be as good as the input data.

Earthquakes in developing countries tend to cause far more damage than in the developed world. This is at least in part because they are not able to implement effective disaster management strategies and strictly enforce building codes, for financial as well as administrative reasons. It is therefore an open question whether planners in local and regional governments in developing countries will have the infrastructure and resources to act on available information on particularly vulnerable regions, for example by structurally reinforcing existing buildings.

Encouraging the involvement of governments of developing nations in the Global Earthquake Model project may be one way of creating greater awareness and a culture of preparedness. Indeed, the project's management intends to solicit active cooperation from scientists from all countries around the world and tap into their expertise on local conditions as well as their access to their governments.

The project is still in its infancy, and it is difficult to gauge its likely impact, let alone evaluate it critically. But it seems to be based on sound foundations: making the tools freely accessible is just, and seeking genuine cooperation of various stakeholders is democratic. The success of the Global Earthquake Model is in everyone's interest; the whole-hearted support of scientists, governments, and the private sector should help it to live up to its promise. 